\title{
New Advances with Next Innovation SurgiGuide: A Practical Insight
}

\author{
${ }^{1}$ Alvaro Farnós Visedo, ${ }^{2}$ Francisco Benet Iranzo \\ ${ }^{1}$ Assistant Professor, Center for Prosthodontics and Implant Dentistry, Loma Linda University, CA, USA \\ Professor, Postgraduate Course of Oral Implantology and Prosthodontics on Implants Technologic Studies Institute \\ San Pablo-CEU University, Madrid, Spain \\ ${ }^{2}$ Associate Professor, Department of Prosthodontics, Cardenal Herrera-CEU University, Valencia, Spain \\ Director, Postgraduate Course of Oral Implantology and Prosthodontics on Implants, Technologic Studies Institutes, San \\ Pablo-CEU University, Madrid, Spain
}

Correspondence: Alvaro Farnós Visedo, Assistant Professor, Center for Prosthodontics and Implant Dentistry, Loma Linda University CA, USA, e-mail: fbenet@ono.com

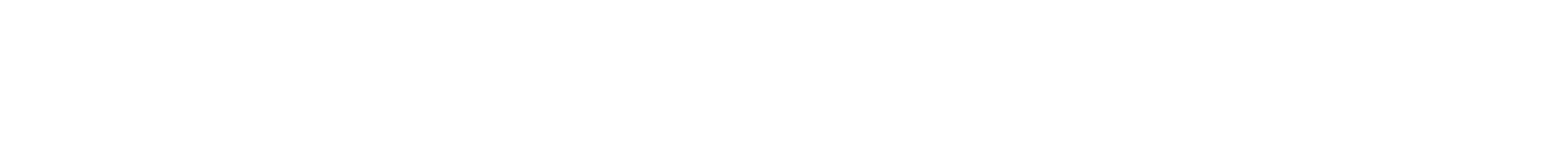

\section{INTRODUCTION}

As part of the dental community, we are all used to the incredibly fast pace in which new and improved products are presented into our clinical practice. The dental companies must keep up with market requirements that are normally not imposed by us clinicians, but by the strong competition between companies and products. This necessity for innovation will often result in advances and products based on marketing and not clinical relevance. As a user of CTbased implant planning software systems since 1999, I have seen my share of these changes that have really not improved anything.

On the other hand Materialize Dental, as a market leader, has been implementing well thought out changes and improvements. The latest set of changes comes with SimPlant 12 and next innovation SurgiGuide: FastTrack and the improved version of SurgiGuide drill guides, which includes the new fixation system for the cylinders in and the labelling of the SurgiGuide drill guides with serial numbers.

The aim of this short paper is to present some of the latest implemented changes and improvements within the company products that I personally find useful in my clinical practice, as well as to illustrate a change in the conventional protocol for SurgiGuide drill guides by placing the implants through the last SurgiGuide drill guide using an internal connection implant driver.

\section{CASE REPORT}

To illustrate everything, a case recently treated in our office will be presented. The patient is a healthy male in his forties wearing an old maxillary partial denture with poor function and esthetics and using retention clasps on teeth 15 and 25. After full diagnosis, an implant-supported milled bar overdenture free in the palatal area was planned as treatment (Fig. 1).

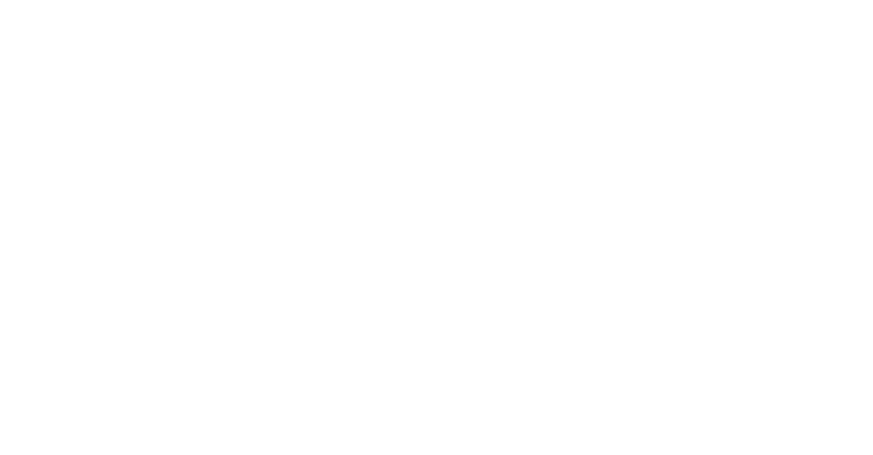

Fig. 1: Existing partial denture in position 


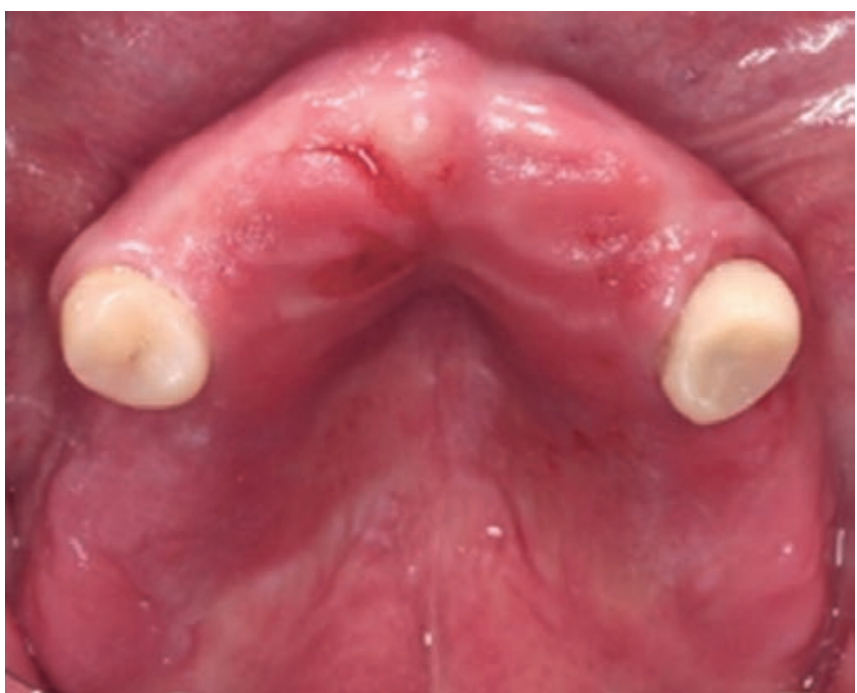

Fig. 2: Occlusal maxillary view

The placement of six implants in the anterior maxilla was decided upon due to bilateral inflammation of the maxillary sinuses. Given the opportunity by Materialize Dental Spain, we decided to try the FastTrack protocol for prompt delivery of the SurgiGuide drill guides (Fig. 2).

In this case, we planned for a mucosa support due to the quantity of bone available in width and height. After duplication of the existing partial denture to fabricate the radiographic scan prosthesis, the patient was sent for CT scan examination on a Thursday. The same day, the data were sent via Internet from the radiology center to the SimPlant conversion center in Madrid. The next morning, the case was ready for virtual surgery on the computer. The six implants were planned, taking the radiographic scan prosthesis and the available bone into consideration. The two posterior implants were planned slightly mesial to the extraction sockets of teeth 15 and 25. A SAFE or Classic SurgiGuide can be chosen. However, to illustrate a more generic protocol for guided implant placement, the Classic SurgiGuide drill guide was ordered for this case (Fig. 3).

A very important step in the process for FastTrack is to let the conversion center know that the case is going to be sent using this new protocol because the SimPlant project must be certified by them. In case of a mucosa-supported SurgiGuide drill guide this only means that the radiographic scan prosthesis has been verified for possible misfit or possible problems with the radiopaque materials in it. You can check if the scan prosthesis is certified in the Toggle 3D objects dialog bottom, right to the 3D window (Fig. 4).

After the case was fully planned, the SurgiGuide drill guide order was sent to Materialize Dental, Belgium on the

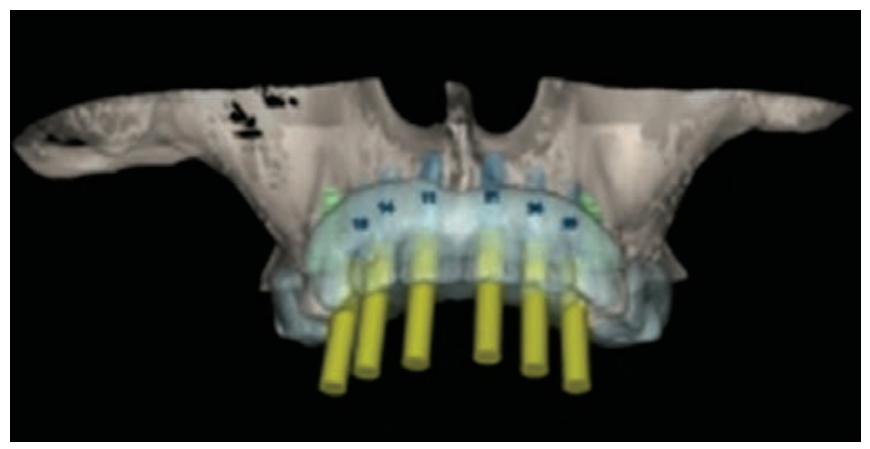

Fig. 3: Planned implants according to space, bone and radiographic scan prosthesis

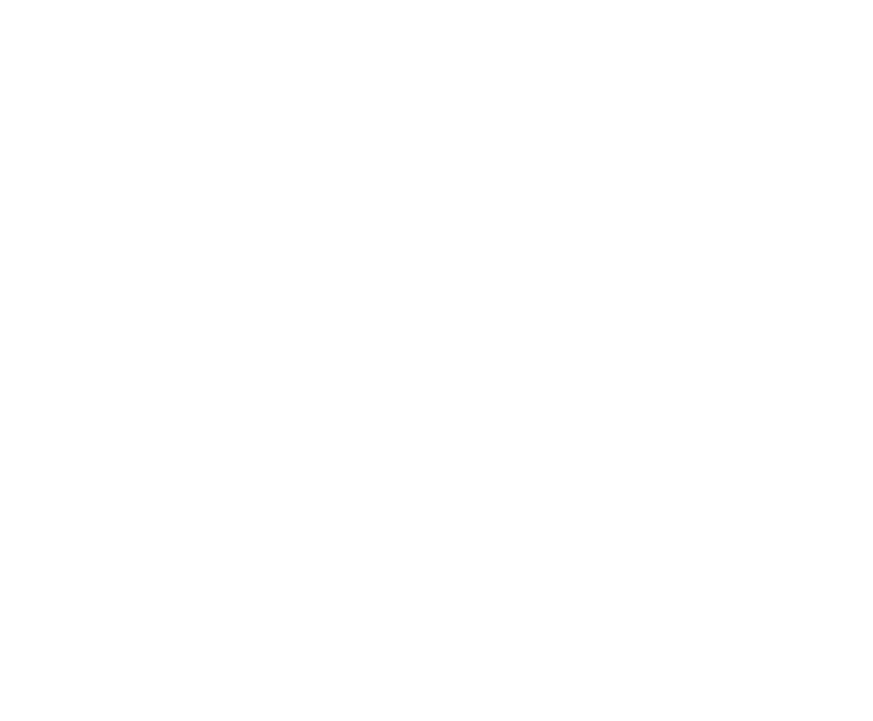

Fig. 4: Three-dimensional objects dialog with the radiographic scan prosthesis certified

following Monday using the request SurgiGuide wizard and the online shop included in the SimPlant 12 version. Thanks to its step-by-step instructions, the request SurgiGuide wizard is extremely easy to use. After a quick registration in the website for the online shop, the SurgiGuide drill guide can be requested FastTrack and after uploading the case your SurgiGuide drill guide order is placed.

The drill guides were received on Friday morning, only 4 full days after order placement. The guaranteed delivery time for FastTrack cases is 2 days faster than for regular cases. The remaining teeth were extracted to be able to try the SurgiGuide drill guides in the patient's mouth (Fig. 5).

Two things can be noticed in Figure 6. Firstly, marked in red, is the number included in the SurgiGuide drill guide which is the patient reference number followed by the number 1, 2 or 3 depending on the position in the sequence of the SurgiGuide drill guide. Secondly, marked in blue, we notice the canals created in the SurgiGuide drill guide going from buccal to palatal and around the metal sleeves. These 


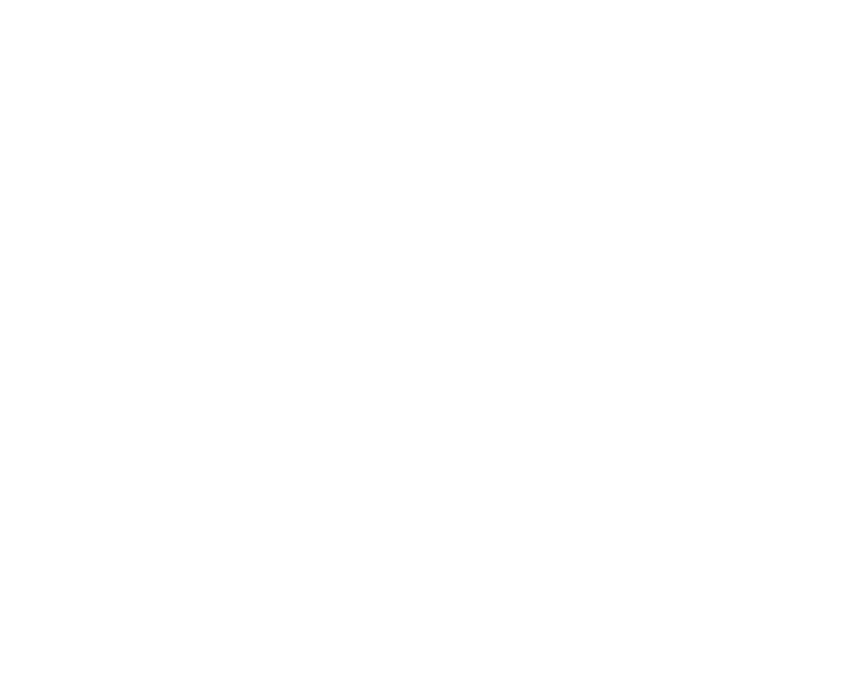

Fig. 5: Occlusal maxillary view after tooth extractions

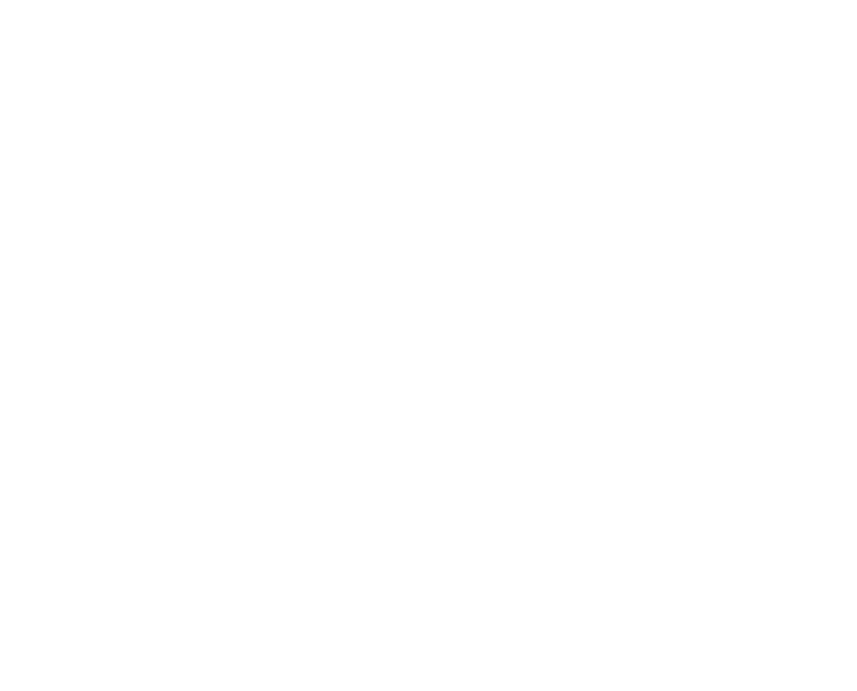

Fig. 6: First template in the SurgiGuide sequence. Serial number (red circle). Canals for adhesive injection (blue circle)

small perforations allow for the injection of the adhesive material and tremendously improve the stability of the metal guiding cylinders within the SurgiGuide drill guide (Fig. 6).

The drilling sequence was followed for the placement of 6 prima implants with a diameter of $4 \mathrm{~mm}$ and a length of $13 \mathrm{~mm}$. For the first SurgiGuide drill guide, the 2.0 drill was used (Fig.7).

The 3.0 drill was used for the second SurgiGuide drill guide. Skipping the last drill is only possible in the maxilla, when the bone is soft enough (Fig. 8).

One of the possible problems with minimally invasive surgery comes at the moment of insertion of the implants. Since the drilling has been done with the SurgiGuide drill guide over the mucosa, we do not have good reference

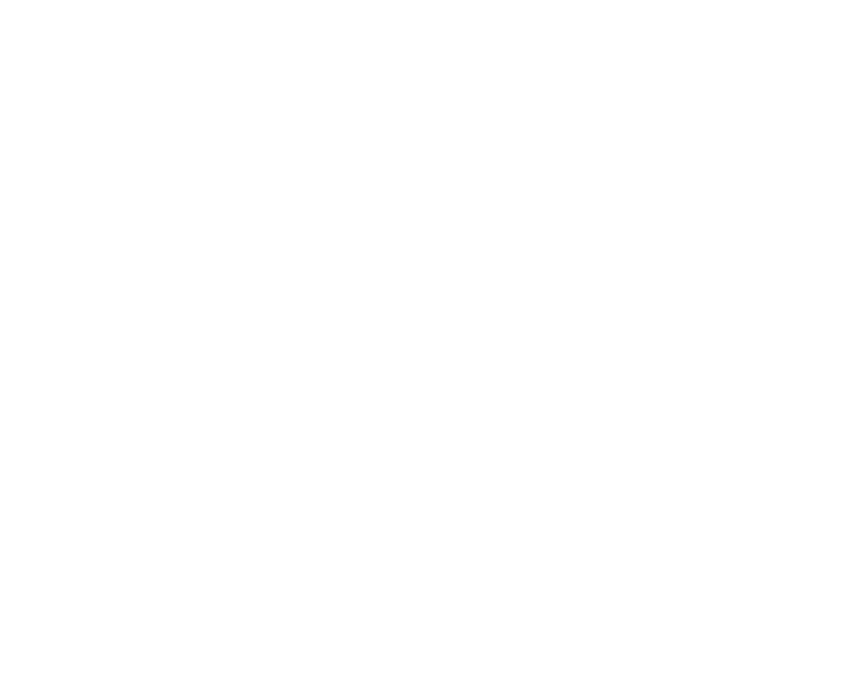

Fig. 7: $2.0 \mathrm{~mm}$ drill taken to final total depth as indicated in the Guideline to SurgiGuide use

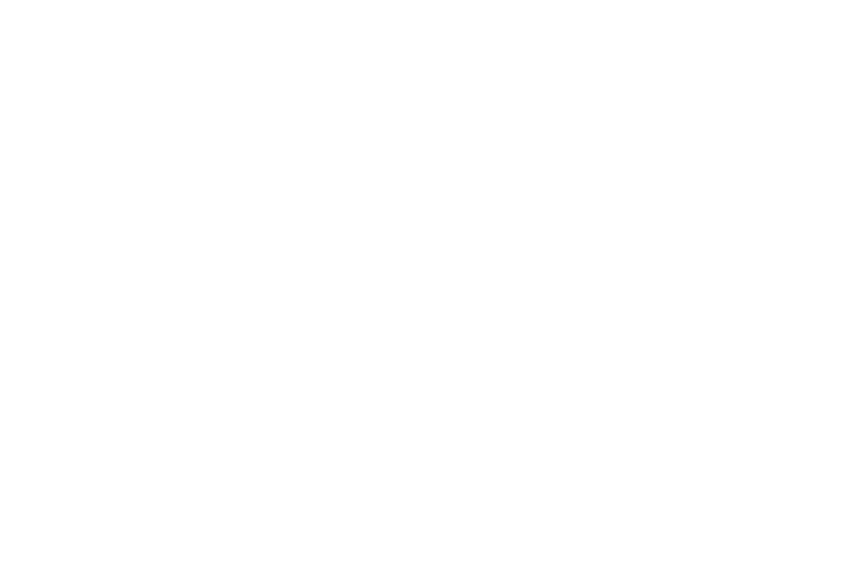

Fig. 8: $3.0 \mathrm{~mm}$ drill through the second SurgiGuide drill guide

points to drive the implants in the correct angulations. This can be easily overcome by placing the implants through a SurgiGuide drill guide (Fig. 9).

Therefore, the last SurgiGuide drill guide can be ordered for a 4.0 drill, but used for insertion of the 4.0 implant. It is also necessary to have an implant driver with the same diameter as the implant itself (Fig. 10).

Unlike the SAFE SurgiGuide drill guide, the Classic SurgiGuide drill guide offers no automatic depth control during insertion of the implants. So after the SurgiGuide drill guide removal, the prosthetic connections have to be placed at the right level using the clinician's experience and periapical radiographs (Fig. 11).

Finally, four healing abutments were placed on the anterior implants (Fig. 12).

The gaps buccal to the posterior implants were filled with xenograft and covered using a collagen plug, and a single crossed suture was given (Fig. 13). 


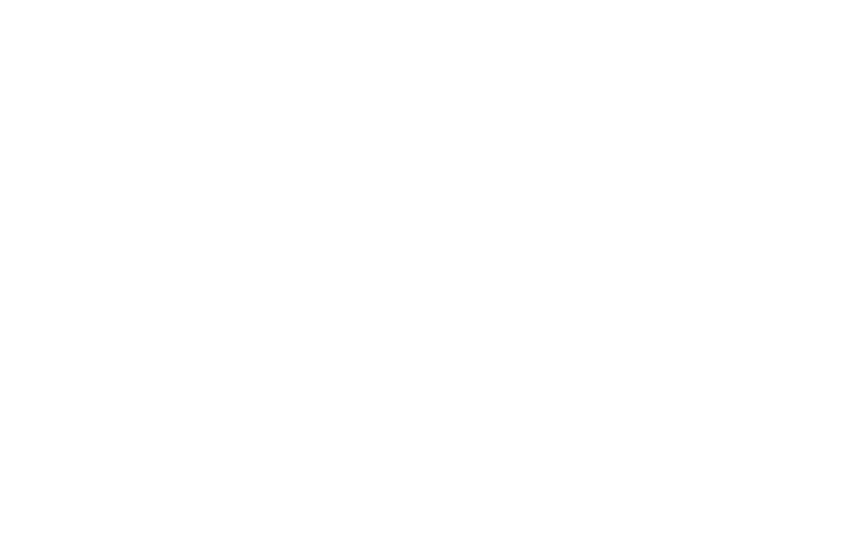

Fig. 9: $4.0 \times 13 \mathrm{~mm}$ implant being driven into position through the third SurgiGuide drill guide

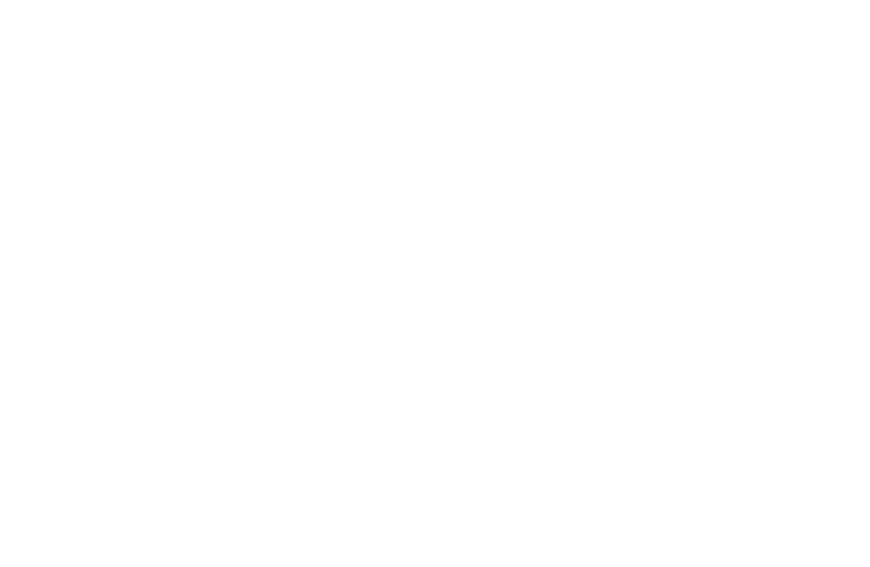

Fig. 10: View of the connections through the SurgiGuide drill guide

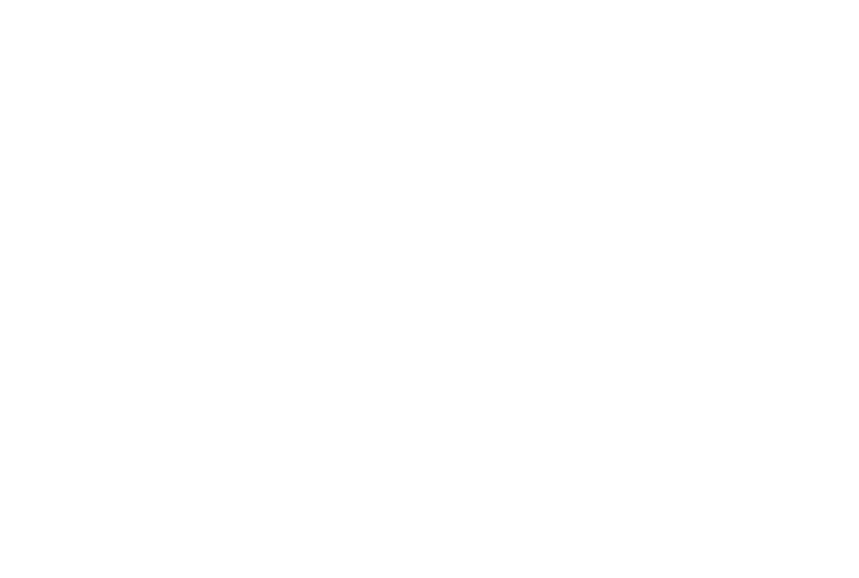

Fig. 11: Occlusal maxillary view with the prosthetic connections prior to final adjustment

\section{CONCLUSION}

A good amount of clinically useful changes have recently occurred within Materialize Dental products: a stronger

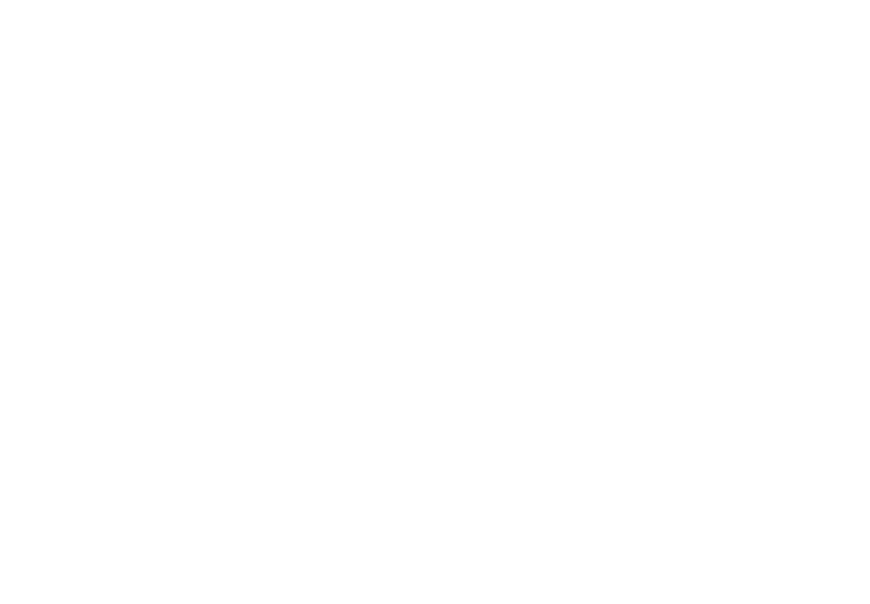

Fig. 12: Healing abutments and sutured extraction sockets

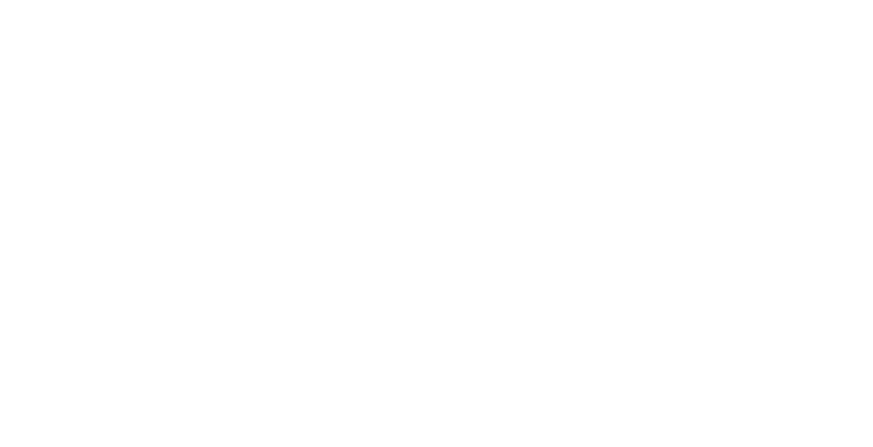

Fig. 13: Postoperative panoramic radiograph for control

fixation of the guiding cylinders in the SurgiGuide drill guides and reference numbers in the drill guides.

The new request SurgiGuide wizard is a very useful tool that allows the surgeon to preview the SurgiGuide drill guide, detect possible problems and change the shape and the design of the drill guide. The latter is only possible for certified SimPlant projects and in case of SAFE and Classic SurgiGuide drill guides. For SAFE SurgiGuide drill guides, you know in advance which drills and implant holders are going to be used, giving you the possibility of change and allowing you to prepare with the right components in advance. Placing the order for the SurgiGuide drill guide online saves time. Additionally, the FastTrack SurgiGuide drill guides are delivered to the clinician in an extremely short period of time.

Moreover, by modifying the SurgiGuide drill guide protocol and placing the implants through the last SurgiGuide drill guide, it is easier to achieve the right angulations for the implants and avoid possible problems for the final prosthesis. This is only possible with implant systems that have the implant driver for the internal connection with the same diameter as the dental implant. 


\section{REFERENCES}

1. Schulze D, Heiland M, Thurmann H, Adam G. Radiation exposure during midfacial imaging using 4- and 16-slice computed tomography, cone beam computed tomography systems and conventional radiography. Dentomaxillofacial Radiology 2004;33:83-86.
2. Guerrero ME, Jacobs R, Loubele M, Schutyser F, Suetens P, van Steenberghe D. State-of-the-art on cone beam ct imaging for preoperative planning of implant placement. Clinical Oral Investigations 2006;10:1-7.

3. David Schneider, Pascal Marquardt, Marcel Zwahlen, Ronald E. Jung A systematic review on the accuracy and the clinical outcome of computerguided template-based implant dentistryClin. Oral Impl Res 2009;20(Suppl. 4):73-86. 